\title{
Retinal break formation following contrecoup trauma: case report
}

\author{
SIMON LONGSTAFF, PAUL S BAINES, AND STUART T D ROXBURGH \\ From the Department of Ophthalmology, Ninewells Hospital, Dundee
}

SUMMARY A previously healthy 38-year-old woman was involved in a road traffic accident resulting in severe commotio retinae. Subsequently extramacular retinal dissolution was noted (not previously described). The patient was treated by closed intraocular microsurgery with a successful anatomical and functional result.

Retinal dissolution at the site of impact is recognised as a complication of coup injuries. Contrecoup injuries have been reported only as leading to retinal hole formation at the macula. This case demonstrates extramacular retinal dissolution following a contrecoup injury (commotio retinae). Internal surgical repair was carried out with a successful result.

\section{Case report}

In June 1984 a previously healthy 38-year-old woman was a front seat passenger in a road traffic accident. She suffered right forehead and upper eyelid lacerations but no skull fracture. She was conscious and orientated and complained of blurred vision in the right eye. As her lacerations were repaired under general anaesthesia her eye was also examined.

Her pupil was noted to be slightly dilated but regular. The fundus showed extensive posterior pole commotio retinae extending just above the superotemporal arcade, 2 disc diameters nasal to the disc and inferiorly to the equator. No retinal breaks were apparent.

Postoperatively she developed diplopia secondary to a right sixth nerve palsy. This rapidly resolved, with no permanent clinical abduction deficit. After seven days her visual acuity was $6 / 9$ and her commotio retinae rapidly resolving.

She was discharged and seen for review elsewhere. She was referred back in April 1985 having noticed in the previous months floaters and a superior, irregularly shaped scotoma slowly encroaching on fixation.

Fundus examination showed two large and one small closely related breaks approximately two disc

Correspondence to Dr Simon Longstaff, Department of Ophthalmology, Royal Hallamshire Hospital, Sheffield S10 2JF.

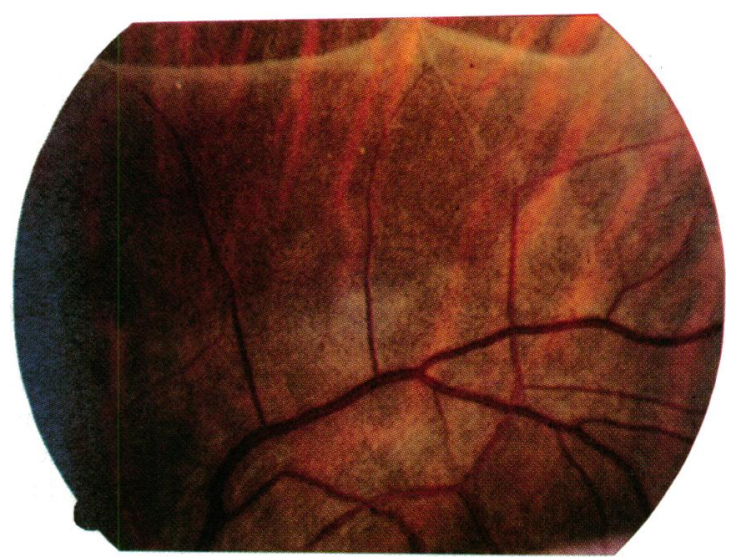

Fig. 1 Preoperative fundal view showing the relation between the upper part of the retinal breaks and the inferotemporal arcade, with subretinal fluid extending under the inferotemporal arcade. (The third small break is not shown in this view.)

diameters below the inferotemporal arcade (Fig. 1). The edges were shallowly elevated, and subretinal fluid extended under the inferotemporal arcade. Her visual acuity was $6 / 6$ and her macula attached. There was no apparent posterior vitreous detachment, and preoperative ultrasound could not demonstrate the posterior vitreous face.

Because of the location and size of the holes an internal repair was carried out, consisting of ocutome vitrectomy, internal tamponade with an air/fluid exchange, and, in the absence of endophotocoagulation, external cryotherapy. The postbasal cortical gel remained in situ, and $360^{\circ}$ cryotherapy was applied to the postoral retina.

Her postoperative course was uneventful. Her 


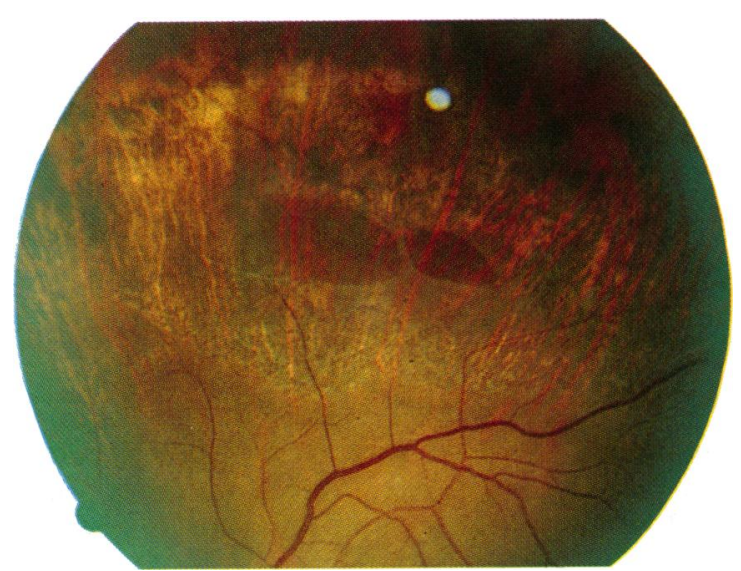

Fig. 2 Postoperative fundal view showing full extent of retinal breaks and surrounding cryoreaction.

visual acuity remains normal and her fundus shows a good cryoreaction below the inferotemporal arcade (Fig. 2). No peripheral breaks were visualised at the time of or subsequent to surgery.

\section{Discussion}

Large irregular retinal breaks have been recognised as a complication of blunt trauma at the point of impact. ${ }^{1-4}$ They are often associated with choroidal rupture, retinal, vitreous, and choroidal haemorrhage, and later onset of choroidal and retinal pigment epithelium atrophy. ${ }^{25}$ These breaks are evident soon after injury, ${ }^{25}$ lead to retinal detachment, and have been attributed to acute retinal necrosis and mechanical disruption. ${ }^{56}$

Commotio retinae is a contrecoup injury' involving the outer retinal layers which macroscopically become opaque. ${ }^{89}$ It may resolve rapidly with a corresponding increase in visual acuity. ${ }^{4}$ It may also be associated with minor pigment epithelium disturbances, particularly at the macula, and may lead to macular hole formation. ${ }^{12}{ }^{10}$ This case shows large areas of dissolution in an area of the posterior pole previously noted to have commotio retinae. The time between break formation and injury is longer than that associated with breaks resulting from direct traurha, and although fluorescein angiography was not carried out there appears to be no choroidal or pigment epithelial involvement.

Large retinal breaks resulting from coup injuries are well documented, but not as a result of a countrecoup (commotio retinae) injury. The mode of injury, with the right forehead and upper eyelid involvement, and in the absence of damage to the inferior orbital margin, suggests a contrecoup injury. A small amount of traumatic mydriasis is known to be associated with contrecoup fundal changes. ${ }^{1+}$

Experimental animal studies have shown commotio retinae to consist of acute photoreceptor outer segment disruption with no extracellular oedema. This is followed by immediate loss of photoreceptor cell bodies in the outer nuclear layer, the permanent outcome being loss of photoreceptor cells. ${ }^{8}$ The final visual outcome depends on the amount of macular photoreceptor cell loss. Severe loss will lead to lamellar or full thickness hole formation because of the absence of other tissue, apart from glial processes, in this area. As extramacular areas are composed of more retinal layers, widespread photoreceptor cell loss alone should not lead to hole formation.

The cause of retinal dissolution in this case is most likely multifactorial. In addition to widespread photoreceptor cells loss there may be loss of inner retinal layers and vitreoretinal interactions leading to hole formation. $360^{\circ}$ cryotherapy was carried out as a prophylaxis against giant tear formation as the postbasal cortical gel remained intact."

\section{References}

1 Archer DB, Canavan YM. Contusional eye injuries: retinal and choroidal lesions. Aust J Ophthalmol 1983; 11: 251-64.

2 Cox MS, Schepens CL, Freeman HM. Retinal detachment due to ocular contusion. Arch Ophthalmol 1966; 76: 678-85.

3 Cox MS. Retinal breaks caused by blunt non-perforating trauma at the point of impact. Trans Am Ophthalmol Soc 1980; 78: 414-66.

4 Eagling EM. Ocular damage after blunt trauma to the eye. $\mathrm{Br} \mathrm{J}$ Ophthalmol 1974; 58: 126-40.

5 Bloome MA, Ruiz RS, Russo CE, et al. Acute retinal necrosis. Ann Ophthalmol 1979; 11 : 723-8.

6 Duke-Elder SW. System of ophthalmology. St Louis: Mosby, 1972; 14: 63.

7 Benson WE. Effects of blunt trauma on the posterior segment of eye. Trans Pa Acad Ophthalmol Otolaryngol 1984; 37: 26-33.

8 Hart JCD, Frank HJ. Retinal opacification after blunt nonperforating concussional injuries to the globe. Trans Ophthalmol Soc UK 1975; 95: 94-100.

9 Sipperley JO, Quigley HA, Gass DM. Traumatic retinopathy in primates. Arch Ophthalmol 1978; 96: 2267-73.

$10 \mathrm{Jacob}$ JSH. Ocular concussion injuries. Trans Ophthalmol Soc $N Z$ 1979; 31: 40-2.

11 McLeod D. Giant retinal tears after central vitrectomy. Br J Ophthalmol 1985; 69: 96-8.

Accepted for publication 8 July 1986. 